\title{
Improvement of verbal fluency in patients with diffuse brain injury over time
}

This article was published in the following Dove Press journal:

Neuropsychiatric Disease and Treatment

24 June 2014

Number of times this article has been viewed

\section{Ana Luiza Zaninotto' \\ Vinícius Monteiro \\ de Paula Guirado² \\ Beatriz Baldivia' \\ Monica Domiano Núñes' \\ Robson Luis \\ Oliveira Amorim² \\ Manoel Jacobsen Teixeira ${ }^{2}$ \\ Mara Cristina Souza \\ de Lucia' \\ Almir Ferreira de Andrade ${ }^{2}$ Wellingson Silva Paiva ${ }^{2}$ \\ 'Division of Psychology, Hospital das Clínicas, ${ }^{2}$ Division of Neurosurgery, University of São Paulo Medical \\ School, São Paulo, Brazil}

Correspondence: Wellingson Silva Paiva University of São Paulo Medical School, 470 Alves Guimarães St, Ap 93,

São Paulo-SP 05410-000, Brazil

$\mathrm{Tel}+55$ II $266 \mid 7226$

Fax +55 II 25486906

Email wellingsonpaiva@yahoo.com.br
Background: Diffuse axonal injury (DAI), a common cause of neurological sequelae in patients with traumatic brain injury (TBI), is considered one of the most prevalent forms of primary neuronal injury in patients with severe TBI. Cognitive deficits induced by DAI can persist over time, especially following moderate or severe injuries. The aim of the present study was to compare verbal fluency (VF) performance at 6 and 12 months after the trauma in a same group of patients with DAI.

Methods: Eighteen patients with moderate to severe DAI and 17 healthy volunteers were enrolled. All DAI participants had sustained a TBI at least 6 months prior to the start of the study, were between 18 and 50 years of age, and had at least 4 years of education. The VF test was administered within an extensive neuropsychological test battery. We evaluated the same patients at 6 months (DAI1 group) and 12 months (DAI2 group) and compared the results of neuropsychological tests with a control group of healthy volunteers who were matched to patients for sex, age, and educational level.

Results: In comparison to controls, the DAI1 group produced significantly fewer words. The DAI2 group produced significantly more semantic words than DAI1 $(P<0.05)$ and demonstrated a trend towards the production of more clusters for letter $\mathrm{A}(P=0.09)$ and total words generated in a phonemic test $(P=0.09)$. No significant differences were observed between DAI2 and the control group in the total number of words generated in phonetic FAS or semantic fluency scores.

Conclusion: The present findings may be useful in the construction of a management plan for long-term TBI rehabilitation that considers the trauma of each patient. Further, our results suggest the VF test is a suitable instrument for the assessment of cognitive difficulties following TBI.

Keywords: traumatic brain injury, neuropsychological tests, diffuse axonal injury, prognosis, executive function

\section{Introduction}

Diffuse axonal injury (DAI), a common cause of neurological sequelae in patients with traumatic brain injury (TBI), is considered one of the most prevalent forms of primary neuronal injury in patients with severe TBI. ${ }^{1,2}$ Head injuries typically induce transient cognitive deficits; ${ }^{3}$ however, these aberrations may persist following moderate or severe injuries. Cognitive processes that may be affected by TBI include mental flexibility, attention, set-shifting, planning, organization, sequencing, judgment, verbal fluency (VF), working memory, and impulsivity. ${ }^{4}$

VF tests commonly used for neuropsychological assessment in both clinical and research settings assess executive function and the spontaneous production of words under restricted search conditions (verbal association fluency). As such, VF tests are valuable tools in the neurological evaluation of individuals with brain damage. ${ }^{5}$ Phonemic fluency is considered reflective of executive function because 
it requires a capacity for verbal retrieval and recall, self-monitoring, effortful self-initiation, and response inhibition. Optimal fluency refers to the ability to generate words within a subcategory and, when a subcategory is exhausted, switch to a new subcategory. These processes are referred to as clustering and switching. ${ }^{6,7}$ Clustering is believed to depend on adequate temporal lobe function, as temporal lobectomy ${ }^{8}$ and Alzheimer's disease ${ }^{9}$ patients exhibit clustering deficits. Conversely, clustering appears to be unaffected by focal frontal lesion. ${ }^{8}$ Switching requires sufficient cognitive flexibility to redirect focus from one subcategory to another and is suggested to be mediated by the frontal lobe. Troyer et $\mathrm{al}^{9}$ observed switching deficits in patients with prefrontal cortex and left superior medial frontal lobe damage that resulted from Parkinson's disease, ${ }^{9,10}$ Huntington's disease, ${ }^{11}$ multiple sclerosis, ${ }^{10}$ or schizophrenia. ${ }^{12}$ Although patients with expansive brain damage exhibit deficiencies in both clustering and switching, frontal focal lesions are significantly associated with a marked reduction in total words generated and total number of switches on both phonemic and semantic assessments. ${ }^{6,8}$ It was further demonstrated that focal temporal lesions also resulted in significant reduction in total words generated and number of switches on a semantic fluency measure. ${ }^{8}$

It has been suggested that more pronounced phonemic fluency (retrieving words that begin with a certain letter) impairments may exist in individuals with frontal lobe damage, whereas semantic fluency (retrieving words that belong to a given semantic category) deficiencies might be more prevalent in individuals with temporal lobe brain damage. ${ }^{13}$

In patients with DAI, impaired executive function is thought to mediate deficiencies in all cognitive capacities: speed, efficiency, and integration of mental processes. ${ }^{5}$ In a recent study, Zakzanis et $\mathrm{al}^{14}$ evaluated switching and clustering components in patients with severe TBI, and observed that VF component scores more accurately reflected severe TBI than the semantic fluency portion of the Controlled Oral Word Association Test. The same pattern was observed in patients with mild TBI. ${ }^{15}$
While previous studies have yielded insight into the performance of brain-damaged patients on VF tests, none has examined the longitudinal performance in patients with traumatic axonal injury. Cognitive deficits induced by brain injury can persist over time, especially following moderate or severe injuries. ${ }^{3}$ The aim of the present study was to compare VF performance at 6 and 12 months after the trauma in a same group of patients with DAI.

\section{Materials and methods}

A prospective study with a 12-month follow-up of 18 patients who had suffered traumatic DAI was conducted. This project was approved by the Institutional Ethics Committee for Research of our institution with register 0097/11, and by the special ethics and research committee of our hospital division of psychology with register 18/2010.

\section{Participants}

The patient group consisted of 18 moderate to severe DAI patients. Moderate to severe DAI in the present study was defined as a Glasgow Coma Scale (GCS) score below 13. All participants had sustained a TBI at least 6 months prior to the start of the study, had experienced loss of consciousness for at least 30 minutes as a consequence of the TBI, were between 18 and 50 years of age, and had at least 4 years of education. Participants were excluded from the study if they had suffered more than one TBI, exhibited evidence of abnormalities other than DAI via 3 Tesla MRI (eg, contusions, epidural hematoma, subdural hematoma), or had been diagnosed with neurological (eg, epilepsy, strokes, tumor) or psychiatric conditions (eg, bipolar disorder, major depression, other conditions requiring admission to a psychiatric ward). The presence of depression symptoms was assessed in patients using the Beck Depression Scale. The control group was paired by age and education (Table 1). Participants were recruited by the neuropsychologist, invitation through caregivers or spouses, or through calls made to hospital staff. Participant recruitment in both groups was conducted in accordance with institutional research guidelines.

Table I Demographic information and verbal fluency performance of healthy controls and DAI patients

\begin{tabular}{lllll}
\hline Variable & Controls $(\mathbf{n}=\mathbf{I 7})$ & & DAl $(\mathbf{n}=\mathbf{l} \mathbf{8})$ \\
\cline { 2 - 4 } & Mean & (SD) & Mean & (SD) \\
\hline Age (years) & 32.17 & $(7.94)$ & 27.05 & $(9.13)$ \\
Education (years) & 9.7 & $(2.88)$ & 10 & $(3.01)$ \\
Sex (male \%) & 65 & & 83.3 \\
\hline
\end{tabular}

Abbreviations: DAI, diffuse axonal injury; SD, standard deviation. 


\section{Procedure}

The fluency test (FT) was administered within an extensive neuropsychological test battery. Participants were asked to provide as many words as possible within 1 minute each letter that began with F, A or S (phonemic test or FAS test) or that were part of a single category (semantic test). Participant responses were written verbatim, with errors or repetitions subsequently excluded from the total score.

Phonemic fluency was determined by tallying the number of words beginning with $\mathrm{F}, \mathrm{A}$, and $\mathrm{S}$ that were generated in 1 minute. Instructions were as follows:

I want you to say as many words as you can that begin with a certain letter. You may say any word, except for proper names like names of persons or places or conjugate nouns or verbs. For example, if you say fazer (do), do not say fazendo (doing) or feito (done).

One point was given for each word said correctly. The total phonologic FT score was the total of all correct words said for each of the three letters (F, A, S).

Semantic fluency was assessed by determining the number of words participants were able to generate in 1 minute that pertained to a single category (animals). One point was given for each animal name that was said correctly. Words inflected in both masculine and feminine forms (eg, galogalinha [rooster-hen]) were counted as one while names of an animal type and associated offspring were counted as separate words (eg, boi-vaca [bull-cow]). Credit was not given to names of subcategories, like birds or fish, if they were specific names within that category (eg, papagaio, andorinha [parrot, swallow]). The total semantic FT was the sum of all correct words.

\section{Clustering and switching}

Consistent with Troyer et $\mathrm{al}^{6}$ repetitions and mistakes, words inflected in both masculine and feminine forms, names of subcategories, and conjugate nouns or verbs were not included in the scoring of clustering and switching.

\section{Phonemic clustering}

A response was considered a phonemic cluster when two consecutive words shared the first consonant and vowel (fala, face) or two consonants and vowel (fralda, franja).

\section{Semantic clustering}

A response was deemed a semantic cluster when animals of the same habitat were listed and classified into relevant subcategories: safari animals, pets, farm animals, insects, reptiles, fish, and birds.

\section{Switching}

Switching was defined as the ability to shift efficiently to a new subcategory (eg, between clusters, cluster-word, wordword which could not form a cluster, both phonemic and semantic FT).

\section{Statistical analysis}

A Student's $t$-test for independent samples was conducted to compare the effect of group on the number of correct words generated in VF tasks and on the number of switches. A matched pair $t$-test analysis was used to compare test performance between groups in tests with a normal distribution. A nonparametric Mann-Whitney test was used to compare the distribution of mean errors and clustering among the groups while a Wilcoxon test was adopted to compare the DAI1 group and DAI2 group (same sample 12 months after the brain trauma).

\section{Results}

The sample consisted of 18 patients $(28.8 \pm 8.8$ years old and $9.35 \pm 2.41$ years of education) and 17 matched healthy controls. Table 1 provides basic demographic and clinical characteristics of the DAI groups, and Table 2 shows the distribution of the type and severity of the trauma and the assessment period for each patient.

There were no significant differences between DAIl and DAI2 groups in the Beck Depression Scale. The descriptions of VF performance of patients and controls are shown in Table 3.

Regarding the FAS FT and trauma severity (assessed by scores on the Glasgow Coma Scale), we found no statistically significant difference in the proportion of improvement in 6 months (DAI1) and 12 months (DAI2) after trauma $(P=0.23)$. When we analyzed the proportion of improvement according to age of the patients, we did not observe statistically significant differences $(P=0.407)$.

\section{Phonemic fluency verbal test measures}

In comparison to controls, an independent samples $t$-test revealed that the DAI1 group produced significantly fewer words in response to letter $\mathrm{A}, t(32)=2.45 ; P<0.05$, and significantly fewer total words on the FAS test, $t(31)=2.1$; $P<0.05$. Comparing the control group with DAI2, we found no significant difference regarding the generation of words in response to letter A, $t(33)=1.7 ; P=0.09$. Also, we found no difference when we compared the results of the DAI1 and DAI2 groups in the generation of total number of words on the FAS test, $t(15)=-1.83 ; P=0.08$. 
Table 2 Distribution of the type and severity of the trauma and assessment period for each patient

\begin{tabular}{lllll}
\hline Patients & Trauma mechanism & Glasgow & $\begin{array}{l}\text { DAll (days } \\
\text { after trauma) }\end{array}$ & $\begin{array}{c}\text { DAI2 (days } \\
\text { after trauma) }\end{array}$ \\
\hline 1 & Motorcycle accident & 3 & 218 & 346 \\
2 & Motorcycle accident & 7 & 161 & 435 \\
3 & Motorcycle accident & 6 & 173 & 446 \\
4 & Car accident & 4 & 182 & 412 \\
5 & Motorcycle accident & 8 & 166 & 437 \\
6 & Fall & 6 & 187 & 453 \\
7 & Motorcycle accident & 8 & 201 & 385 \\
8 & Motorcycle accident & 5 & 160 & 377 \\
9 & Motorcycle accident & 6 & 213 & 378 \\
10 & Motorcycle accident & 9 & 185 & 354 \\
11 & Car accident & 7 & 179 & 473 \\
12 & Car accident & 9 & 223 & 384 \\
13 & Being run over & 6 & 223 & 389 \\
14 & Being run over & 10 & 174 & 390 \\
15 & Motorcycle accident & 6 & 227 & 388 \\
16 & Fall & 8 & 178 & 423 \\
17 & Car accident & 6 & 182 & 422 \\
18 & Motorcycle accident & 5 & 211 & 397 \\
\hline
\end{tabular}

Abbreviations: DAI, diffuse axonal injury; DAII, 6-month evaluation; DAI2, 12-month evaluation.

No significant group differences were found in mean phonemic errors, mean switches, and clustering.

\section{Semantic fluency verbal test measures}

The DAI2 group produced significantly more semantic words than DAI1, $t(17)=-2.90 ; P<0.05$. We additionally observed a trend in the control group towards higher average semantic fluency than the DAI1 group.

Moreover, sample comparison revealed that the control group performed significantly more switches than the DAI1 group, $t(33)=2.27 ; P<0.05$.

No significant differences were observed between DAI2 and the control group in the total number of words generated in phonetic FAS test $(P=0.24)$ or semantic fluency scores $(P=0.48)$.

\section{Discussion}

The present study demonstrated that TBI patients produced fewer words in the phonological and semantic subsets of the $\mathrm{VF}$ test at 6 months posttrauma relative to controls. However, overall improvement was observed at 12 months posttrauma in the total number of words produced, especially in the phonological VF test. Thus, 6 months after the injury, the patients performed worse than the control group in variables such as total number of generated words and number of switches. Moreover, there was no difference between the performance of the control group and the DAI2 group in any of the analyzed variables, suggesting VF improves over time.

These findings indicate that DAI patients exhibit a different cognitive profile over time, characterized by improved information processing; however, there was no difference

Table 3 Verbal fluency performance of healthy controls and DAI patients

\begin{tabular}{|c|c|c|c|c|c|c|c|}
\hline Fluency test & Variable & $\begin{array}{l}\text { Controls } \\
(n=17)\end{array}$ & (SD) & $\begin{array}{l}\text { DAII } \\
(n=18)\end{array}$ & (SD) & $\begin{array}{l}\text { DAI2 } \\
(n=18)\end{array}$ & (SD) \\
\hline \multirow[t]{4}{*}{ Phonemic } & Words generated & 27.4 & $(9.1)$ & 21.1 & $(8)$ & 24.1 & $(6.8)$ \\
\hline & Mean switches & 18.9 & $(8.3)$ & 16.1 & $(7.5)$ & 17.9 & $(5.1)$ \\
\hline & Mean cluster size & 1.76 & (I.3) & 1.19 & (I) & 1.94 & (1.3) \\
\hline & Total errors & 3.6 & $(2.5)$ & 4.9 & $(3.7)$ & 4.5 & (3) \\
\hline \multirow[t]{4}{*}{ Semantic } & Words generated & 15.4 & $(4.7)$ & 12.8 & $(4.5)$ & 14.4 & (3.7) \\
\hline & Mean switches & 10.6 & $(4.7)$ & 7.4 & $(3.8)$ & 9.2 & $(3.8)$ \\
\hline & Mean cluster size & 1.5 & $(1.3)$ & 1.3 & (I) & 1.4 & (I) \\
\hline & Total errors & 0.24 & $(0.4)$ & 0.39 & $(0.8)$ & 0.44 & $(0.6)$ \\
\hline
\end{tabular}

Abbreviations: DAI, diffuse axonal injury; DAII, 6-month evaluation; DAI2, 12-month evaluation; SD, standard deviation. 
between measures that assessed aspects of executive functions, such as cluster and switches. The significantly lower number of switches in the DAI1 group relative to controls and the increase in VF phonological clusters over time (DAI1 versus DAI2) suggests a neural reorganization. Bashir et $\mathrm{al}^{16}$ suggest that neuroplasticity occurs in patients after mild trauma, as cortical pathophysiology associated with postconcussion syndrome abates after approximately 6 weeks.

VF tests are notoriously sensitive to the measurement of executive functions in various types of patients, including patients with TBI. ${ }^{17,18}$ These tasks measure the brain's ability to access lexical stores, retrieve information from semantic memory, and switch cognitive set. ${ }^{7,13,19}$ It is assumed that patients with TBI exhibit impairments on FTs because of executive function deficiencies, particularly in processes related to self-generative behavior. ${ }^{20} \mathrm{TBI}$ leads to greater deficits in phonemic rather than in semantic fluency, probably due to its impact on the frontal lobes. ${ }^{21-23}$ Frontal lobe dysfunction is implicated in these impairments despite the fact that TBI often causes diffuse damage and temporal lobe damage. ${ }^{24}$

Contrary to the observation ${ }^{14}$ that components of the VF test (clusters and switches) were more sensitive measurements of executive function in DAI patients than the total word number, our study demonstrated that the total number of generated words was the most important component of both the phonological and semantic tests. The previous study ${ }^{14}$ showed that the semantic VF test was more effective at differentiating between the TBI and control groups. In our study of a specific group of patients with brain injury, we observed that the phonological VF test significantly differentiated between the groups during the first evaluation period (6 months posttrauma) but not at the second evaluation period (12 months posttrauma). During the second evaluation, the performance of the DAI group did not differ significantly from that of controls. This improvement over time may be due to neuroplastic activity that follows brain injury and the effect of test learning. ${ }^{23}$

While Bercaw et $\mathrm{al}^{24}$ stated that recovery from TBI deficits is expected by 6 months posttrauma, some authors contest $^{25,26}$ that such patients do not return to premorbid skill levels. In the present study, we observed a time-dependent performance improvement in the total number of words generated; however, measures of clustering and switching do not seem to have been as sensitive in the Brazilian population as the total number of words generated in the tests. This finding contrasts with those of studies that evaluated populations of TBI patients in other regions, such as Canada and Israel, ${ }^{14,17}$ and may be because of the small sample size of the DAI group. The present findings may be useful in the construction of a management plan for long-term TBI rehabilitation that considers the trauma of each patient. Further, our results suggest the VF test is a suitable instrument for the assessment of cognitive difficulties following TBI.

\section{Disclosure}

The authors report no conflicts of interest in this work.

\section{References}

1. Andrade AF, Paiva WS, Amorim RL, Figueiredo EG, Rusafa Neto E, Teixeira MJ. [The pathophysiological mechanisms following traumatic brain injury]. Rev Assoc Med Bras. 2009;55(1):75-81. Portuguese.

2. Andrade AF, Paiva WS, Amorim RL, et al. Continuous ventricular cerebrospinal fluid drainage with intracranial pressure monitoring for management of posttraumatic diffuse brain swelling. Arq Neuropsiquiatr. 2011;69(1):79-84.

3. Lima DP, Simão Filho C, Abib Sde C, de Figueiredo LF. Quality of life and neuropsychological changes in mild head trauma. Late analysis and correlation with S100B protein and cranial CT scan performed at hospital admission. Injury. 2008;39(5):604-611.

4. Lezak MD, Howieson DB, Loring DW, Hannay HJ, Fischer JS. Neuropsychological Assessment. 4th ed. New York: Oxford University Press; 2004.

5. Henry JD, Crawford JR. A meta-analytic review of verbal fluency performance following focal cortical lesions. Neuropsychology. 2004;18(2):284-295.

6. Troyer AK, Moscovitch M, Winocur G. Clustering and switching as two components of verbal fluency: evidence from younger and older healthy adults. Neuropsychology. 1997;11(1):138-146.

7. Troyer AK. Normative data for clustering and switching on verbal fluency task. J Clin Exp Neuropsychol. 2000;22(3):370-378.

8. Troyer AK, Moscovitch M, Winocur G, Leach L, Freedman M. Clustering and switching on verbal fluency tests in Alzheimer's and Parkinson's disease. J Int Neuropsychol Soc. 1998;4(2):137-143.

9. Troyer AK, Moscovitch M, Winocur G, Alexander MP, Stuss D. Clustering and switching on verbal fluency: the effects of focal frontaland temporal-lobe lesions. Neuropsychologia. 1998;36(6):499-504.

10. Tröster AI, Fields JA, Testa JA, et al. Cortical and subcortical influences on clustering and switching in the performance of verbal fluency tasks. Neuropsychologia. 1998;36(4):295-304.

11. Rich JB, Troyer AK, Bylsma FW, Brandt J. Longitudinal analysis of phonemic clustering and switching during word-list generation in Huntington's disease. Neuropsychology. 1999;13(4):525-531.

12. Robert PH, Lafont V, Medecin I, et al. Clustering and switching strategies in verbal fluency tasks: comparison between schizophrenics and healthy adults. J Int Neuropsychol Soc. 1998;4(6):539-546.

13. De Capitani EM, Vieira RJ, Madureira PR, et al. Auditory neurotoxicity and hepatotoxicity after MSMA (monosodium methanarsenate) high dose oral intake. Clin Toxicol (Phila). 2005;43(4):287-289.

14. Zakzanis KK, McDonald K, Troyer AK. Component analysis of verbal fluency scores in severe traumatic brain injury. Brain Inj. 2013;27 (7-8):903-908.

15. Zakzanis KK, McDonald K, Troyer AK. Component analysis of verbal fluency in patients with mild traumatic brain injury. J Clin Exp Neuropsychol. 2011;33(7):785-792.

16. Bashir S, Vernet M, Yoo WK, Mizrahi I, Theoret H, Pascual-Leone A. Changes in cortical plasticity after mild traumatic brain injury. Restor Neurol Neurosci. 2012;30(4):277-282.

17. Kavé G, Heled E, Vakil E, Agranov E. Which verbal fluency measure is most useful in demonstrating executive deficits after traumatic brain injury? J Clin Exp Neuropsychol. 2011;33(3):358-365. 
18. Mathias JL, Bigler ED, Jones NR, et al. Neuropsychological and information processing performance and its relationship to white matter changes following moderate and severe traumatic brain injury: a preliminary study. Appl Neuropsychol. 2004;11(3):134-152.

19. Kavé G. Phonemic fluency, semantic fluency, and difference scores: normative data for adult Hebrew speakers. J Clin Exp Neuropsychol. 2005;27(6):690-699.

20. Busch RM, McBride A, Curtiss G, Vanderploeg RD. The components of executive functioning in traumatic brain injury. J Clin Exp Neuropsychol. 2005;27(8):1022-1032.

21. Jurado MA, Mataro M, Verger K, Bartumeus F, Junque C. Phonemic and semantic fluencies in traumatic brain injury patients with focal frontal lesions. Brain Inj. 2000;14(9):789-795.

22. Vakil E. The effect of moderate to severe traumatic brain injury (TBI) on different aspects of memory: a selective review. J Clin Exp Neuropsychol. 2005;27(8):977-1021.
23. Wilson BA, Watson PC, Baddeley AD, Emslie H, Evans JJ. Improvement or simply practice? The effects of twenty repeated assessments on people with and without brain injury. J Int Neuropsychol Soc. 2000;6(4): 469-479.

24. Bercaw EL, Hanks RA, Millis SR, Gola TJ. Changes in neuropsychological performance after traumatic brain injury from inpatient rehabilitation to 1-year follow-up in predicting 2-year functional outcomes. Clin Neuropsychol. 2011;25(1):72-89.

25. Dikmen SS, Machamer JE, Powell JM, Temkin NR. Outcome 3 to 5 years after moderate to severe traumatic brain injury. Arch Phys Med Rehabil. 2003;84(10):1449-1457.

26. Dikmen SS, Corrigan JD, Levin HS, Machamer J, Stiers W, Weisskopf MG. Cognitive outcome following traumatic brain injury. J Head Trauma Rehabil. 2009;24(6):430-438.

\section{Publish your work in this journal}

Neuropsychiatric Disease and Treatment is an international, peerreviewed journal of clinical therapeutics and pharmacology focusing on concise rapid reporting of clinical or pre-clinical studies on a range of neuropsychiatric and neurological disorders. This journal is indexed on PubMed Central, the 'PsycINFO' database and CAS, and is the official journal of The International Neuropsychiatric Association (INA). The manuscript management system is completely online and includes a very quick and fair peer-review system, which is all easy to use. Visit http://www.dovepress.com/testimonials.php to read real quotes from published authors. 